\title{
Multilayer distributed model predictive control of urban traffic
}

\author{
G. B. Castro, J. S. C. Martini \& A. R. Hirakawa \\ São Paulo University, Brazil
}

\begin{abstract}
Urban traffic is a relevant topic for the population of cities that suffer from daily traffic jams. People not only spend time on their journeys but also lose energy. With the purpose of improving the traffic flow, it is possible to control the temporization of traffic lights. However, because of the intrinsic characteristics of the system - multivariable, stochastic and dynamic - controlling the traffic of a city through the temporization of its actuators is insufficient to achieve an optimal level. This way, the objective of this work is to elaborate a multilayer distributed model predictive control (ML-DMPC) of the traffic. The proposed model is composed of two layers of control: local and global. Each agent of control is responsible for the local control logic of a set of interdependent semaphores - in other words, semaphores that belong to the same intersection between streets. In order to achieve the local control objective, each control agent is modelled as a neural network and the following components are weighted: the waiting time of the vehicles; the waiting time of the pedestrians; and synchronization of the semaphores. The input variables of the system - flow of vehicles and pedestrians in all directions - are treated by fuzzy logic for the purpose of improving the quality of the information. The principles of consensus between control agents and behavioural coordination are used to conciliate local and global control objectives. In the global control layer, reinforcement learning is applied to improve the modelling process of complex dynamic systems, as the city traffic, as well as to provide adaptability to the model through learning. By means of the proposed model, it is possible to achieve the dynamic control of the traffic lights system of a city.

Keywords: multilayer control, model predictive control, urban traffic control, distributed control, artificial intelligence, interconnected dynamic systems.
\end{abstract}




\section{Introduction}

A portion of the citizens of urban centers spend significant time daily in traffic. Urban mobility becomes even more complicated when there is a growth in a city's population that is not accompanied by an improvement in its infrastructure. This way, the citizens spend more time than necessary during daily commuting due to traffic jams. These traffic jams are generated by a higher number of vehicles in the streets than the city is capable of receiving. Not only is the traffic system unable to adapt to the quantity of vehicles and people in the streets, but also, in many cases, it doesn't offer better options of commuting.

Semaphores are used in order to organize the flow of vehicles. In the conventional way, they are controlled through preprogrammed time counters. As a consequence, the green time of each semaphore is determined according to past analysis in a trial to better manage the traffic. However, the traffic system is complex due to its dynamic behavior, which generates a different flow pattern of vehicles each day, and not deterministic - in other words, random events, such as heavy rains, accidents or flooding, influence its behavior. Therefore, the conventional control is only sufficient to organize the flows, but not optimal. Studies in the area of urban traffic show alternative methods with the purpose of improving the control efficiency.

Karakuzu and Demirci [1] developed a fuzzy logic controller with the purpose of minimizing the global waiting time at an intersection. The controller was compared to a conventional semaphore controller and the simulations demonstrated that the statistic approach to model the system results in a closer behavior to the real traffic. Instead of using a controller with fixed green times of each semaphore, they used a comparison of the length of the vehicle queues in each street to set the green times adaptively. In spite of having a better control performance, the control parameters were determined beforehand, which has as a consequence a less flexible control. Moreover, the proposed method handles only one intersection, which is a considerable simplification of the dynamics of the traffic.

Oliveira and Camponogara [2] modeled the dynamic system as a multiagent system with the purpose of decomposing a centralized control problem in various sub problems of distributed control. The main advantages are the scalability and the adaptability of the system, which makes it flexible to changes - an intrinsic part of its behavior. Thus, when a new semaphore is added to the system, it is not necessary to reprogram the control of the whole system. The focus in [2] is to model the connection of the agents in the distributed system. Some principles used by Oliveira and Camponogara [2] are used as the foundation of this work, such as the Distributed Model Predictive Control (DMPC). However, in order to increase even more the adaptability of the system, we propose, in this work, a second control layer. The second control layer is the possibility of enabling the model to consider the dynamic nature of the global system. In other words, in case the system changes due to accidents or to permanent infrastructure changes, there will be no need to reprogram the model in each control agent, because they will be able to identify these changes and adapt themselves. 
The distribution of the semaphores control is also proposed by Gibaud et al. [3]. The principle of auto organization is adopted through wireless communication between vehicles to better estimate the traffic behavior. The cooperation model proposed in this work enable a faithful prediction of the system's states. Furthermore, they proposed a strategy to reduce the communication necessity without affecting the quality of the auto organization. Despite being an alternative that models the nonlinear traffic dynamics with precision, the practical deployment of such a strategy is complicated because of the required process of acquiring information.

[4-6] use image processing techniques to monitor the traffic and enable a more efficient control due to the higher quantity of available information. Choudekar [4] control the traffic in a reactive way, using the images to determine the vehicle density in a street and adjust the green time of the semaphore based on it. Kumar et al. [5] develops vehicle behavior recognition in order to predict its trajectory. Afif [6] uses fuzzy logic in order to determine the green time of the semaphores. The method consists in trajectory estimation based on predictions made through image processing. The use of information extracted from video cameras shows improvement in the control efficiency due to the new set of input parameters. Thereby, predicting the behavior of the vehicles is an efficient way of adapting the semaphore control in order to avoid a purely reactive control.

The distribution of the control capacity, as in [2, 3], and the consequent division of the problem in sub problems is the principle of the proposed control model. This method, as described by Antonelli [7], has different ways of approaching the control problem. We use the principle of consensus between agents - Antonelli [7] and Moreau [8] - as a way of modeling the interaction between neighbors and ensuring the local control optimum. Besides, based on the principle of behavior coordination - addressed by Antonelli [7], Brooks [9] and Mataric [10] - we divided the control problem in two layers: local and global. Therefore, it is possible to define a global control objective and conciliate it with the local control objectives. Thus, we use an artificial intelligence technique, reinforcement learning, in order to better define the global dynamic of the system and approach the global control optimum.

The principles of consensus and behavior coordination are used to establish weak connections between agents. This way, the system becomes more flexible and, in case an agent fails, the whole system is not compromised, as pointed out by Oliveira and Camponogara [2]. The focus of this work is the structure of the proposed model and the interaction between the local and global control layers. It is noticeable that other works don't explore the presence of the pedestrians, whose influence in the traffic dynamic is significant. Their behavior differs to the behavior of the vehicles in the following aspects: cluster density; locomotion speed; flow linearity.

The following section of this work consists of the model proposal. The third section concludes this work and elucidates possible future works. 


\section{Model proposal}

The proposed model is constituted of two parts: the local control layer and the global control layer. The Multilayer Model Predictive Control (ML-DMPC) is represented through the diagram in Figure 1. A comparison with the centralized - represented in the upper left corner - and distributed control - represented in the lower left corner - methods facilitate the comprehension of how it works.

Each control agent consists of a set of semaphores that control the flows of vehicles and pedestrians at an intersection. The interaction between neighboring agents and the internal dynamics of each control agent belong to the local layer. Meanwhile, the global vision of the system and the capacity of adapting the control model are the main characteristics of the global layer.

The local control layer determines the behavior of the internal states of a control agent, which controls a set of independent semaphores - in other words, the semaphores that belong to the same street intersection. The duty cycles of the semaphores of an intersection can be modeled through a neural network in order to handle the multiple inputs and multiple outputs of its nonlinear dynamic. The fuzzy logic is used to improve the quality of the inputs, which are stochastic and dynamic.

The interaction between neighboring control agents is modeled through the Distributed Model Predictive Control, as done by Oliveira and Camponogara [2]. This way, it is possible to conciliate the control objectives of the neighbors, which are in the majority, contradictory, favoring a common global objective. The exchange of information between neighbors is essential to enable the traffic behavior prediction.

The second control layer consists of the action of the control agents regarding a global objective control - in this case, the minimization of the total waiting time of vehicles and pedestrians in the city. To achieve that, we based the control decision on the principle of behaviour coordination and on an artificial intelligence method; reinforcement learning. Thus, it is possible to evaluate the local control decisions of the agents and redefine their rules as necessary. In order to do so, the global control layer analyse the critical situations in the city traffic and, after the causes are determined, the algorithm assigns new weights to the control parameters of the agents involved. The main objective of this control layer is to reduce the criticality of punctual situations and hence approximate the performance measure function to the global optimum. Therefore, it is possible to provide a global adaptability to the model and enable a better control behaviour regarding the control of nonlinear dynamic systems.

The following subsections handle the control layers with more details.

\subsection{Local control layer}

The local control layer can be divided by its two features: the internal dynamics of the control agent; and the interaction between control agents. As the focus of this work is not the model of the local control layer, we present an explanation of how it works, but don't give details of its mathematical equations. 


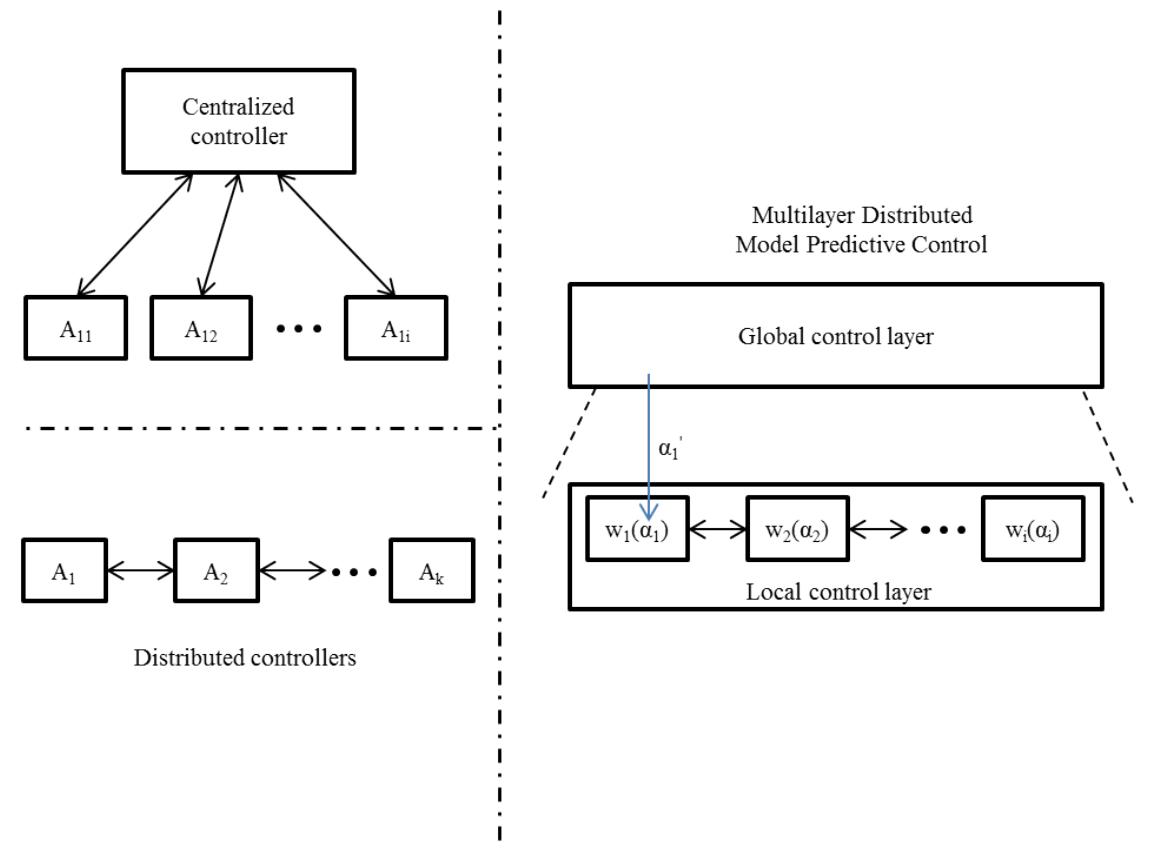

Figure 1: Centralized control, distributed control and multilayer distributed model predictive control.

The internal dynamics of the control agent is represented in Figure 2. The inputs of the system are handled by fuzzy logic in order to simplify its stochastic nature. An example of its advantages is the vehicle density input. Thereby, nonlinearities in the density pattern can be ignored and the relative occupancy of the streets - the information that really matters - becomes the new input to the neural network. As a multiple input multiple output (MIMO) nonlinear system, the traffic can be modeled using a neural network, as done by Srinivasan et al. [11]. Hence, it is possible to model not only the relationship between the inputs and outputs, but also the complex couplings between state variables.

The interaction between neighbors is based on a DMPC, Camponogara et al. [12]. Thus, the neural network model of the traffic is used to determine the relationship between inputs and outputs and enable a measurement of performance. The basic principle of a Model Predictive Control (MPC) method is to optimize an objective function. As the traffic is modeled as a distributed system, we also need to split this objective function into multiple objective functions, one for each control agent, as done by Camponogara and Oliveira [13]. This way, it is possible to use the state variables of each control agent in order to determine the best control strategy through an optimization technique. A similar strategy - the combination of a neural network and a MPC method - was used by Pan and Wang [14]. 
Neural Network

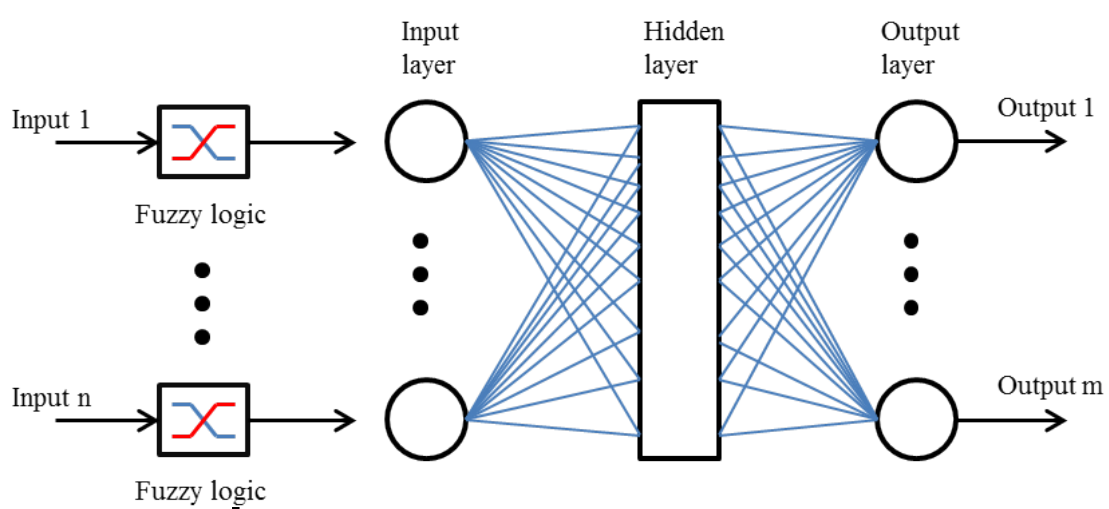

Figure 2: $\quad$ Control agent.

\subsection{Global control layer}

The main contribution of this work is the addition of a global control layer. Its objective is to provide a global vision, long term adaptability and a better control performance to the system. A similar approach was done by Puig et al. [15] in drinking water networks, but with a different purpose. Their purpose was to increase the importance of the global control objective of the system, in order to better conciliate it with the local objectives, and consequently improve the control performance.

The global control layer is a centralized control loop with a larger operation time interval. This larger time interval consists of the time required for the global controller to acquire information of all control agents. Thus, the global control layer has a global vision of the system, which enables a global analysis of critical situations that the local agents cannot perceive due to the complexity of the stochastic global dynamic and to the limited information received by them. Therefore, the system can not only react adaptively in a short time scale, such as fractions of a second, but also react to global changes in the system model in a longer time scale, such as minutes.

In order to achieve the desired adaptability, the global controller interacts with the control agents through the neural network. After acquiring the information about the state variables of all the control agents, the global controller uses a heuristic search to determine the most critical situations. This way, it can determine which control agents are involved in these situations and adapt their behavior to avoid it. As the control agents interact only with their neighbors, they can cause traffic jams without perceiving it. This happens because the state variables of all control agents are coupled. But, in order to 


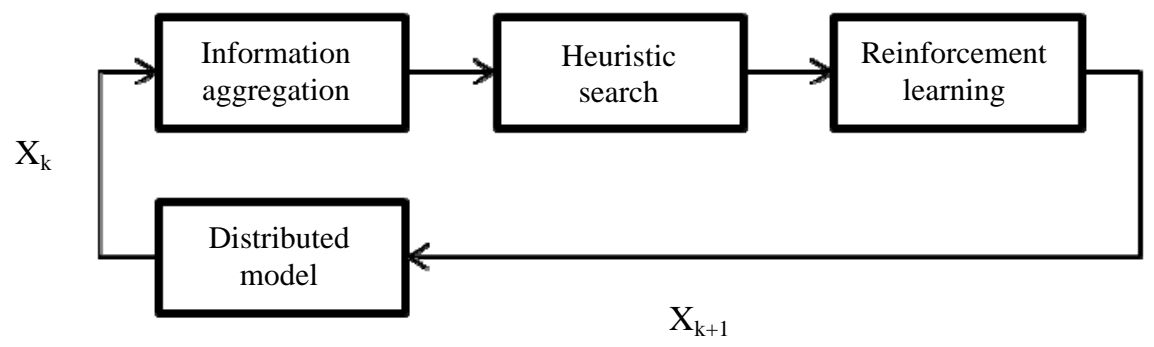

Figure 3: Global control layer.

achieve distributed control, the multiagent method decouples them as a simplification hypothesis. In spite of having a satisfactory local control performance, the complexity of nonlinear, stochastic dynamic systems prevents these control strategies to achieve a real global optimum. At the same time that the novel proposed model keeps the weak connections between control agents, which also have its advantages, as enlightened by Oliveira and Camponogara [2], it uses a longer operation cycle to provide an indirect global vision and adaptability to them. Therefore, it is possible to achieve a better control performance globally.

The interaction of the global controller with the control agents occurs through the weights of the neural networks that model the dynamic of the traffic. In a neural network, the artificial neurons have weighted connections between each other. The initial weights are provided by the traffic model of the local agents and can be changed by the global controller according to a reinforcement learning method. The block diagram represented in Figure 3 elucidates the operation steps of the proposed global control layer. The purpose of the recalculations of these parameters is to adapt the model to changes in its original dynamics and to provide a higher priority to one of the flows, in case a semaphore is responsible for a chain effect that it cannot perceive.

The reinforcement learning method is based on occasional rewards and modifies the agent's decision mechanisms to improve its control performance. The conventional approach of a reinforcement learning method consists of providing to an agent a model of the world - where the agent acts - based on rewarding it for good decisions and penalizing it for bad ones. This way, after a learning time, the agent creates a model of the world itself. A typical problem of this approach is the convergence of the algorithm or, in other words, if it can achieve a final model that is consistent with the reality. In our case, the convergence is not an issue, because we use reinforcement learning to adapt a model to stochastic situations and not to create it. Eqn (1) represents how the method updates the weights of the neural networks:

$$
X_{k+1}=(1-\alpha) X_{k}+\alpha r,
$$


where $r$ is the reward, $\alpha$ is the learning rate and $X$ is the updated variable - the weights of the neural networks, in this case. The reward and the learning rate can be chosen freely and regards, respectively, the intensity and the speed with which the model adapts itself to changes. $X_{k}$ is the representation of the matrix of weights $W$ of the neural networks in a given time $k$, represented in eqn (2). Each connection between artificial neurons has a given weight $w_{i j}$ and the variable update in the reinforcement learning method doesn't need to change all of them through the rewards - only those which need more or less priority.

$$
W=\left[\begin{array}{ccc}
W_{11} & \cdots & w_{1 j} \\
\vdots & \ddots & \vdots \\
w_{i 1} & \cdots & w_{i j}
\end{array}\right]
$$

where $\mathrm{w}_{11}$ is the weighting coefficient that multiplies the first connection of the first artificial neuron, $\mathrm{w}_{\mathrm{i} 1}$ is the weighting coefficient that multiplies the $\mathrm{i}^{\text {th }}$ connection of the first artificial neuron, $w_{1 j}$ is the weighting coefficient that multiplies the first connection of the $\mathrm{j}^{\text {th }}$ artificial neuron and $\mathrm{w}_{\mathrm{ij}}$ is the weighting coefficient that multiplies the $\mathrm{i}^{\text {th }}$ connection of the $\mathrm{j}^{\text {th }}$ artificial neuron.

\section{Conclusion and future work}

The elaboration of a model which aims to avoid traffic jams more efficiently affects not only the citizens by saving their time, but also the economy and the environment. An improvement in the traffic flow reduces the time and the fuel spent by citizens, reducing, as a consequence, economical expense and environmental impact. Furthermore, traffic jams retard the action of emergency services, such as fire trucks and ambulances. Indirectly, the time and energy spent can even be accounted as health and economic production losses.

The conventional centralized control methods are not suitable for large scale systems, such as urban traffic. This problem occurs in many other areas, such as robotics, biology and economics. Therefore, a big scientific effort is focused on researching distributed control methods. However, there are still many open problems in this relatively new field. The specific problem treated in this work, a distributed nonlinear dynamic system with multiple inputs and multiple outputs, has many issues to be approached. The one approached in this work provides an improvement regarding global adaptability and control performance through an additional control layer.

Future work includes the simulation of a real scenario in order to evaluate the behavior of the proposed model. Thereby, it will be possible to analyze the consequences of varying different control parameters of the global control layer, such as the learning rate and the reward. Simulations can also display numerically the discussed advantages and reveal possible limitations of the novel model, which will constitute a new opportunity of research. 
The local control layer is one of the possible future areas of research. One open issue is the short term predictability of the model. The MPC optimization regards mainly the long term prediction of the system. This way, the nonlinear dynamics of the flows of vehicles and pedestrians are not handled properly. Consequently, their time of acceleration and deceleration are not considered and represent a lost time in the present models. The synchronization between neighbors would be sufficient to take these dynamics into consideration. An interesting approach would be to elaborate a dedicated predictive neural network specifically to handle these new state variables and synchronize the green times of neighbors based on their short term predictions.

\section{Acknowledgement}

This research was supported by CNPq, Conselho Nacional de Desenvolvimento Científico e Tecnológico, Brasil.

\section{References}

[1] Karakuzu, C. and Demirci, O., Fuzzy logic based smart traffic light simulator design and hardware implementation. Applied Soft Computing, 10(1), pp. 66-73, 2010.

[2] Oliveira, L.B. and Camponogara, E., Multi-agent model predictive control of signaling split in urban traffic networks. Transportation Research Part C: Emerging Technologies, 18(1), pp. 120-139, 2010.

[3] Gibaud, A., Thomin, P. and Sallez, Y., FORESEE, a fully distributed selforganized approach for improving traffic flows. Simulation Modelling Practice and Theory, 19(4), pp. 1096-1117, 2011.

[4] Choudekar, P., Implementation of Image Processing in Real Time Traffic Light Control. 2011 3rd International Conference on Electronics Computer Technology, eds. S. Banerjee and M.K. Muju, pp. 94-98, 2011.

[5] Kumar, P., Ranganath, S., Weimin, H. and Sengupta, K., Framework for Real-Time Behavior Interpretation From Traffic Video. IEEE Transactions on Intelligent Transportation Systems, 6(1), pp. 43-53, 2005.

[6] Afif, F.A., Enhanced adaptive traffic signal control system using camera sensor and embedded system. 2011 International Symposium on MicroNanoMechatronics, eds. M.F. Rachmadi and A. Wibowo, pp. 367-372, 2011.

[7] Antonelli, G., Interconnected Dynamic Systems: An Overview on Distributed Control, IEEE Control Systems, 33(1), pp. 76-88, 2013.

[8] Moreau, L., Stability of continuous-time distributed consensus algorithms. 43rd IEEE Conference on Decision and Control, pp. 3998-4003, 2005.

[9] Brooks, R.A., A robust layered control system for a mobile robot. IEEE Journal of Robotics and Automation, 2(1), pp. 14-23, 1986.

[10] Mataric, M.J., Behavior-based control: Examples from navigation, learning, and group behavior. Journal of Experimental and Theoretical Artificial Intelligence, 9(2-3), pp. 323-336, 1997. 
[11] Srinivasa, D., Choy, M.C. and Cheu, R.L., Neural Networks for Real-Time Traffic Signal Control. IEEE Transactions on Intelligent Transportation Systems, 7(3), pp. 261-272, 2006.

[12] Camponogara, E., Jia, D., Krogh, B.H. and Talukdar, S., Distributed Model Predictive Control. IEEE Control Systems, 22(1), pp. 44-52, 2002.

[13] Camponogara, E. and Oliveira, L.B. IEEE Transactions on Systems, Man and Cybernetics - Part A: Systems and Humans, 39(6), pp. 1331-1338, 2009.

[14] Pan, Y. and Wang, J., Model Predictive Control of Unknown Nonlinear Dynamical Systems Based on Recurrent Neural Networks. IEEE Transactions on Industrial Electronics, 59(8), pp. 3089-3101, 2012.

[15] Puig, V., Hierarchical Temporal Multi-Layer Decentralized MPC Strategy for Drinking Water Networks: Application to the Barcelona Case Study. $20^{\text {th }}$ Mediterranean Conference on Control and Automation (MED), eds. Ocampo-Martinez, C. and Montes de Oca, S., pp. 740-745, 2012. 